\title{
TmHMM Algorithm
}

National Cancer Institute

\section{Source}

National Cancer Institute. TmHMM Algorithm. NCI Thesaurus. Code C49038.

A computer algorithm that predicts trans-membrane helices based on the hidden Markov model. 